\title{
“Evolution of respiratory function in Duchenne muscular dystrophy from childhood to adulthood." Antonella LoMauro, Marianna Romei, Sandra Gandossini, Riccardo Pascuzzo, Simone Vantini, Maria Grazia D’Angelo and Andrea Aliverti. Eur Respir J 2018; 51: 1701418.
}

\section{CrossMark}

This article from the February 2018 issue of the European Respiratory Journal was originally published with errors in figure $5 \mathrm{~b}$ and $\mathrm{d}$. The shaded areas representing statistical significance in these panels were not presented correctly. The corrected figure is presented below, and the original article has been corrected and republished online. No further changes to the body text of the article were needed to address the error.
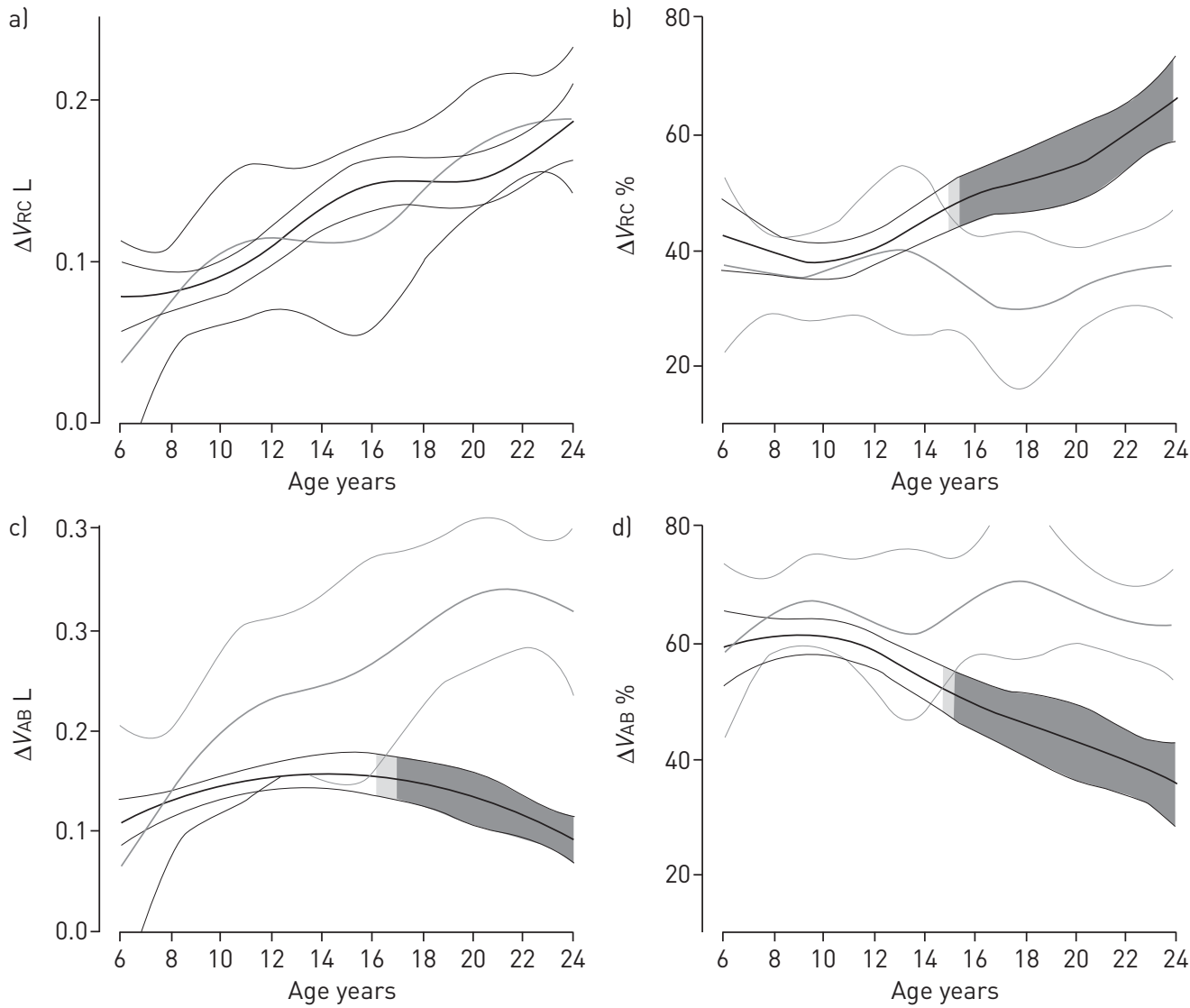

FIGURE 5 Evolution with age of the maximum-likelihood estimation population mean curve (thick line) and its $95 \%$ pointwise asymptotic confidence intervals (thinner lines) of ribcage volume variations ( $\Delta V_{R C}$ ) and abdominal volume variation ( $\triangle V_{\mathrm{AB}}$ ) expressed in litres (a and $c$, respectively) and as percentage contribution to tidal volume ( $b$ and $d$, respectively) during spontaneous breathing in supine position in DMD patients (black lines) and in healthy controls (grey lines). Light grey areas: values significantly different from predicted with $0.01<p<0.05$; dark grey areas: values significantly different from predicted with $p<0.01$. 